\title{
A Miniature Four-Hole Probe for Measurement of Three-Dimensional Flow with Large Gradients
}

\author{
Ravirai Jangir, Nekkanti Sitaram, and Ct Gajanan \\ Thermal Turbomachines Laboratory, Department of Mechanical Engineering, IIT Madras, Chennai 600 036, India \\ Correspondence should be addressed to Ravirai Jangir; raviraijangir90@gmail.com
}

Received 28 May 2014; Revised 11 August 2014; Accepted 12 August 2014; Published 2 September 2014

Academic Editor: Mark McQuilling

Copyright ( 2014 Ravirai Jangir et al. This is an open access article distributed under the Creative Commons Attribution License, which permits unrestricted use, distribution, and reproduction in any medium, provided the original work is properly cited.

\begin{abstract}
A miniature four-hole probe with a sensing area of $1.284 \mathrm{~mm}^{2}$ to minimise the measurement errors due to the large pressure and velocity gradients that occur in highly three-dimensional turbomachinery flows is designed, fabricated, calibrated, and validated. The probe has good spatial resolution in two directions, thus minimising spatial and flow gradient errors. The probe is calibrated in an open jet calibration tunnel at a velocity of $50 \mathrm{~m} / \mathrm{s}$ in yaw and pitch angles range of \pm 40 degrees with an interval of 5 degrees. The calibration coefficients are defined, determined, and presented. Sensitivity coefficients are also calculated and presented. A lookup table method is used to determine the four unknown quantities, namely, total and static pressures and flow angles. The maximum absolute errors in yaw and pitch angles are 2.4 and 1.3 deg., respectively. The maximum absolute errors in total, static, and dynamic pressures are 3.4,3.9, and $4.9 \%$ of the dynamic pressures, respectively. Measurements made with this probe, a conventional five-hole probe and a miniature Pitot probe across a calibration section, demonstrated that the errors due to gradient and surface proximity for this probe are considerably reduced compared to the five-hole probe.
\end{abstract}

\section{Introduction}

The use of the multihole pressure probes has become common to determine total and static pressures, flow velocity, and flow directions in three-dimensional flow fields with suitable calibrations. Multihole pressure probes make accurate and simultaneous measurement of total and static pressures and flow direction when pressure and velocity gradients are small. Pressure probes have some advantages over other methods as their maintenance, relatively low cost, and simplicity in operation. Hence these are preferred in research and industrial purposes. In principle, any aerodynamic body such as cylinder, sphere, wedge, or prism, with a number of holes can be used to measure three-dimensional flows. A minimum of four holes on an aerodynamic body is required to measure the four unknowns, namely, total and static pressures and two angles in mutually perpendicular planes, in three-dimensional flows. However for the sake of symmetry and extended range of measurement capability, five-hole and seven-hole probes are preferred.
Because of their simplicity in operation and low cost, extensive investigations are carried out on multihole probes, particularly on five-hole probes, on their calibration and data reduction methods and their application to complex three-dimensional flow measurements. Treaster and Yocum [1] reported on calibration of different types of five-hole probes and their errors due to Reynolds number variation and surface proximity effects. Pisasale and Ahmed [2] had developed a method to extend the useful operating range for highly three-dimensional flows by replacing the conventionally defined denominator in the calibration coefficients with a more complex denominator. Pissasale and Ahmed $[3,4]$ developed theoretical relationship based on potential flow for calibration and application of five-hole flows. Yasa and Paniagua [5] developed a robust method for five-hole probe calibration. This technique demonstrates that the five-hole probe can be used even if one of the side holes is blocked. Dominy and Hodson [6] investigated the effect of various factors including head shape and Reynolds number on the calibration of five-hole probes. Lee and Jun [7] calibrated a 
commercial five-hole probe at different Reynolds numbers. They found that the effect of Reynolds number on the calibration coefficients is different at different yaw and pitch angles. A comprehensive review of recent developments in multihole probe technology is presented by Telionis et al. [8] Recently Lien and Ahmed [9] have used a five-hole probe to measure skin friction coefficient in complex two and threedimensional flows. This technique avoids the necessity of aligning a Preston probe with the flow direction and the necessity of a wall static tap.

But in three-dimensional flows with large pressure and velocity gradients, in flows such as tip clearance vortex and other complicated flow phenomena that occur in turbomachinery, these probes make erroneous measurements due to their relatively large size.

Spatial errors can be minimized in two ways first by minimizing the probe head dimensions and second by applying corrections. Earlier, work was done on the miniaturization of multihole probes. To characterize Dean's vortices, Ligrani et al. [10] developed a miniature five-hole probe (diameter $1.22 \mathrm{~mm}$ ) and used in low speed channel flow and they applied a correction method to account for spatial errors. However this method has limitations on the size of the pressure tubes that are used to make the probes. Smaller diameter tubes have longer response time. Also the small tubes may be easily blocked by dirt and may give erroneous measurements or may not give any measurements.

The other alternative is to develop methods to correct the measurements for the errors due to pressure and velocity gradients and surface proximity. This approach was adopted by Chernoray and Hjärne [11], Town et al. [12], and Honen et al. [13]. However these methods may require larger number of measurements. For the minimum errors due to pressure and velocity gradients and surface proximity effects, it will be necessary to combine both the techniques.

The probe size is relatively large in the above cases. However the pressure and velocity gradients are large in tip clearance vortex, end wall flows, flow in corner of blades, and other complex flows that occur in turbomachinery. Hence there is a strong requirement of further miniaturisation of the probe head for highly three-dimensional flow measurements. For three-dimensional flow measurements a four-hole probe which can measure four independent pressures can be used.

Four-hole probes have some advantages over five- and seven-hole probes as fewer measurements and reduced instrumentation are required during calibration and application. The measuring volume of the probe head is small compared with the five-hole probe. Hence the spatial errors caused by large pressure and velocity gradients and errors due to the surface proximity effects and shear gradients effects are reduced.

Four-hole probes come in many configurations and are used for many applications. The simple and earliest four-hole probe was obtained by modifying a three-hole cylindrical probe. An additional hole which is mainly sensitive to the flow in the pitch plane is added to the end of a threehole probe. This type of probe is known as cantilevered four-hole cylindrical probe and used in many measurement applications in turbomachinery (Erwin, [14]) and other flows (Maheshwari et al., [15]). Similar four-hole probe with wedge configuration is commercially available from ACflow Corporation [16]. Heneka [17] and Ainsworth et al. [18] developed similar four-hole wedge probes with fast response pressure transducers to measure periodic total and static pressures, velocity and its three components, and flow angles. These probes are usually large about $3 \mathrm{~mm}$ in diameter and the measuring errors due to pressure and velocity gradients and surface proximity are usually large. However it has to be mentioned that Schlienger [19] developed a cantilever cylindrical probe of $1.2 \mathrm{~mm}$ tip diameter with a spatial resolution of about $1 \mathrm{~mm}$. This type of probe is very useful to measure the flows in diffusers of centrifugal compressors and in labyrinth seals of axial turbines, where the spanwise flow angles are usually small. However for many turbomachinery flow measurements, pressure probes with very small measurement volumes and capability to measure large spanwise angles are needed. The available literature on such probes is presented below.

A four-hole probe which satisfies partially the above requirements was developed by Shepherd [20] for the threedimensional flow measurements. The main feature of the probe is a tip shaped like the frustrum of a pyramid, with three-side holes equispaced around a central hole. This probe was calibrated in yaw and pitch angle range of $\pm 40^{\circ}$ and the calibration space is divided into six zones, making the use of this probe somewhat complicated. Sitaram and Treaster [21] have developed and presented two miniature four-hole probes. The probe heads were fabricated from $0.55 \mathrm{~mm}$ outer diameter and $0.30 \mathrm{~mm}$ inner diameter stainless steel hypodermic tubes. The probe heads are machined to a 50 degree half angle cone and located approximately four local support diameters upstream to reduce support interference effects. These probes have slightly less measuring volumes and higher spatial resolution compared to the probe developed by Shepherd. The probe with pyramid head was extensively used in many flow measurement applications.

Based on the above literature the commonly used fourhole probes can be divided into three designs, namely, modified cylindrical/hemi spherical probe, wedge/pyramid probe, and forward facing tube probe. These probes are presented in Figure 1 . Both modified cylindrical/hemi spherical probe and wedge/pyramid probe can be used when the measurement space is limited, such as in diffuser passages of centrifugal compressors and turbomachinery seals. But the pitch angle range of these probes is limited and cannot be increased. A properly designed forward facing tube four-hole probe can be used to measure highly three-dimensional flows with large variations in both yaw and pitch angle ranges.

The forward facing tube probe 2 shown in Figure 1 can be used both in the four-hole and five-hole probe modes. In the four-hole probe mode, tube 5 was not used. Tube 5 could be eliminated or used to mount a thermocouple. This probe has a smaller error due to velocity gradient effect, as the probe height in the pitch plane is equal to two-tube diameters only. The four-hole probe 1 shown in 1 and five-hole probe have larger errors due to velocity gradient effect, as their heights in the pitch plane are two and one-half times tube diameters and three times tube diameters, respectively. It should be 


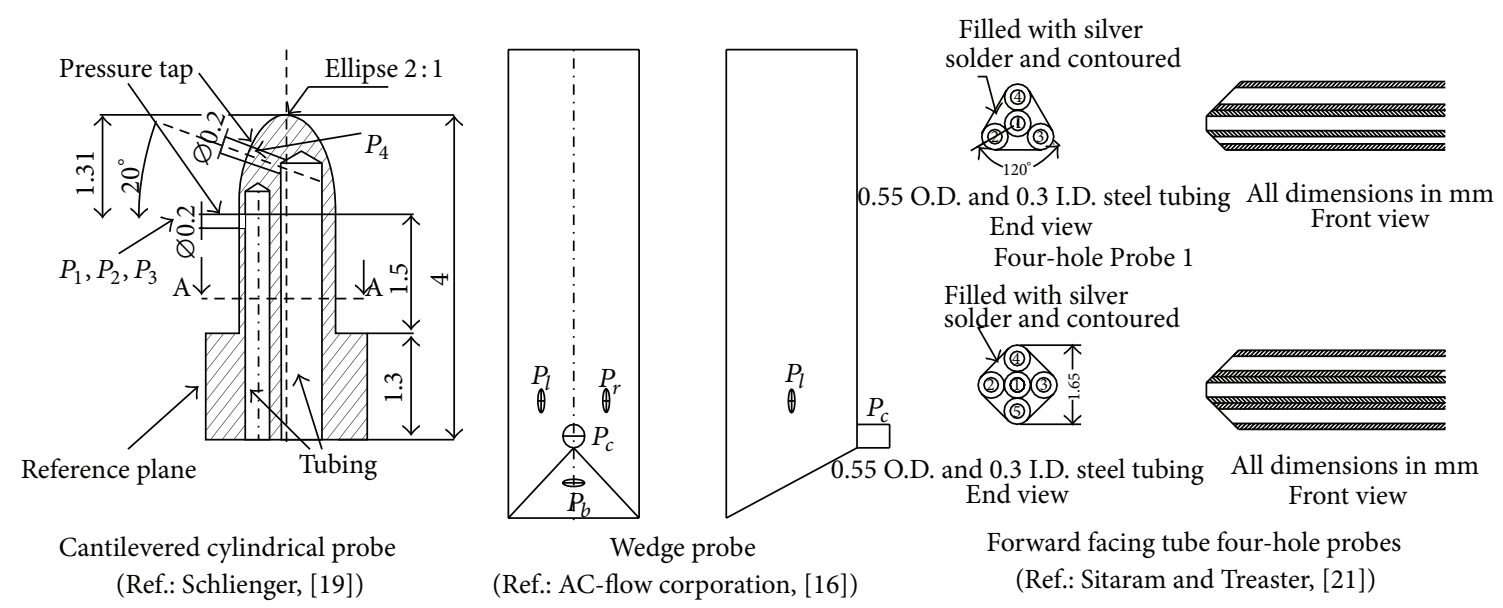

FIGURE 1: Commonly used four-hole probe configurations.

noted that these probes are smaller than any commercially available probes, so that spatial and velocity gradient effects are minimized.

The probes described above have small errors due to pressure and velocity gradients and surface proximity errors. However these errors are still large in many turbomachinery flows. Hence it is necessary to develop methods to further reduce the spatial and surface proximity errors.

The four independent pressures can be related to determine the four unknowns, namely, total and static pressures and two flow angles in mutually perpendicular planes. From the total and static pressures, velocity can be calculated. Using the flow angles, the three velocity components can be calculated. Hence a four-hole probe is chosen for development. Probe tip design can vary diversely depending on the particular application. A pressure probe must be designed for particular application where it is used. Four-hole probes are used by Sitaram and Treaster [21] and other researchers. However the configuration used by these researchers has good spatial resolution in one direction only. A four-hole probe with good spatial resolution in two directions for highly three-dimensional flow measurement is developed in this paper.

\section{Objective}

The objective of the present investigation is to design, fabricate, calibrate, and validate a miniature four-hole probe with minimum spatial error for accurate three-dimensional flow measurement with large pressure and velocity gradients as in cases of tip clearance vortex, flows in corner of blades, end wall flows, and other complex flows that occur in turbomachinery.

\section{Design and Fabrication of Four-Hole Probe}

For three-dimensional flow, four (at least) or more holes strategically located on a probe head are necessary to determine the flow. Design of any multihole probe is a compromise between many conflicting requirements, such as small probe tip versus good response and large yaw and pitch angle measurement capability versus sensitivity of the calibration coefficients. As the size of the probe tip is reduced, by using smaller tubes, the response of the pressure measuring time increases. Hence more time is required for data acquisition. Smaller chamfer angle of the probe gives larger yaw and pitch angle measurement capability at the expense of reduced sensitivity of the calibration coefficients. The present probe is designed to optimise these conflicting requirements. In the present investigation, the four holes correspond to the centre hole, one yaw hole and two pitch holes of a fivehole probe. This design deviates from the design of earlier four-hole probes, which use one centre hole, two yaw holes, and one pitch hole of a five-hole probe. The commonly used five-hole probes are symmetric about both yaw and pitch axes, while the commonly used four-hole probes are symmetric about the yaw axis. The commonly used fourhole probes have more yaw angle range and better sensitivity of calibration coefficients in the yaw plane. The advantage of the present design is that the pitch angle range can be increased, although the yaw angle range is reduced. This is not a major disadvantage as the probe can be easily change its yaw angle when the flow exceeds the calibration yaw angle range. However a new technique needs to be developed to determine reference yaw angle. The centre hole with a chamfer angle of $90^{\circ}$ to the tube gives a measure of total pressure and is denoted by $P_{C}$. The side hole is chamfered at an angle of about $35^{\circ}$ to the yaw plane and is noted as $P_{S}$ and is mainly sensitive to the yaw angle variation. The bottom and top holes are chamfered at an angle of about $35^{\circ}$ to the pitch plane and are noted as $P_{B}$ and $P_{T}$, respectively, and are mainly sensitive to the pitch angle variation. Hence four independent pressures can be measured, which are sufficient to define the threedimensional flow. It has been already demonstrated that small chamfer angle gives higher calibration angle range. Hence small chamfer angle is chosen for the present design.

The probe head design is chosen so as it has good spatial resolution in both yaw and pitch directions. Assuming the tube size is the same, five-hole and seven-hole probes have a 


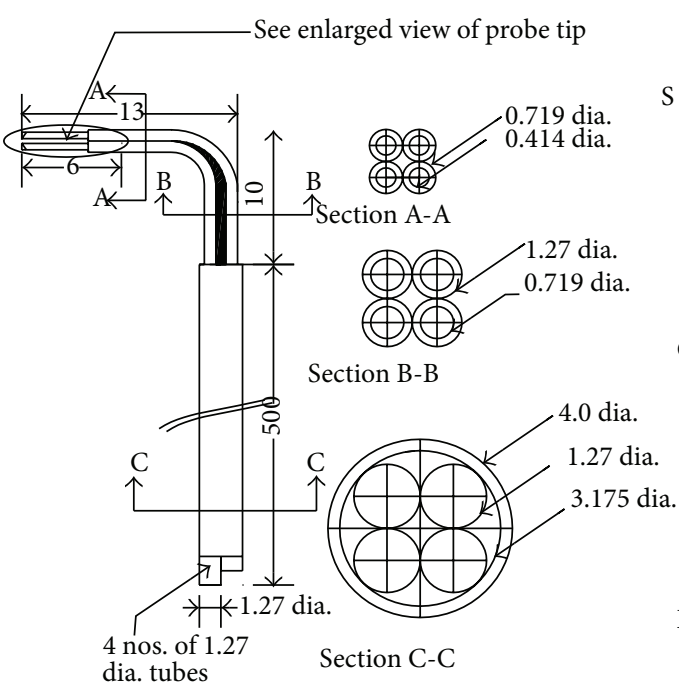

All dimensions are in $\mathrm{mm}$

material: stainless steel

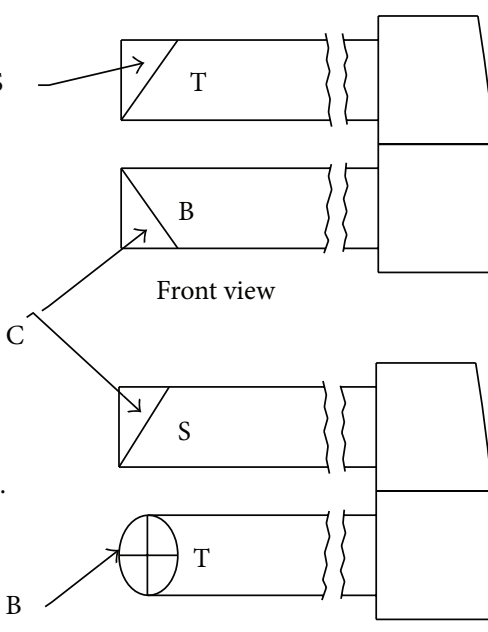

Top view

Enlarged views of probe tip

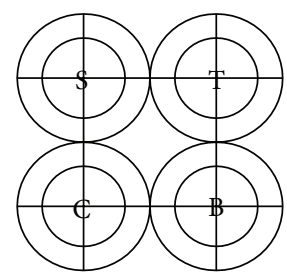

Side view

$\begin{array}{ll}\text { B: bottom hole } & \text { S: side hole } \\ \text { C: centre hole } & \text { T: top hole }\end{array}$

(a)

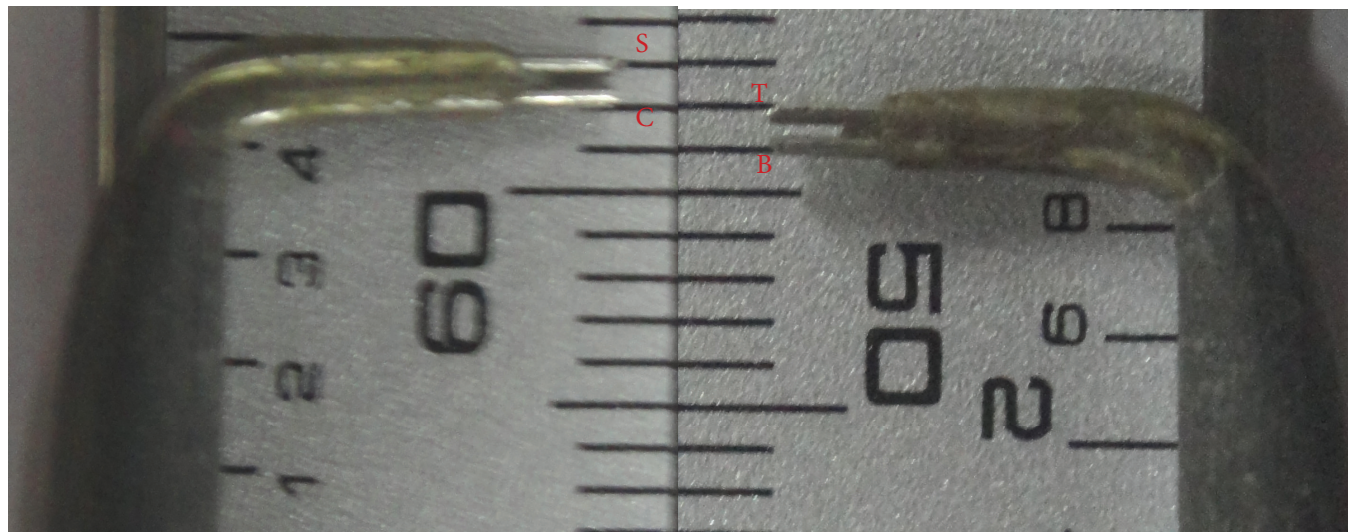

(b)

Figure 2: (a) AutoCAD drawing of probe. (b) Photo of probe tip.

spatial resolution of $3 d$ ( $d$ : tube diameter) in both directions. Four-hole probes of existing designs have a spatial resolution of $3 d \times 2 d$ in yaw and pitch directions. The present probe has a spatial resolution of $2 d$ in both directions. Therefore it can be used for highly three-dimensional flows such as flow in corner of blades and end wall flows with large pressure and velocity gradients in all directions. The probe is L-shaped and the probe head details are shown in Figures 2(a) and 2(b). The material of the all tubes used in fabrication of four-hole probe is stainless steel. The final probe has a measuring area of $1.284 \mathrm{~mm}^{2}$. Design and fabrication details and the sizes of hypodermic tubes used for fabrication of the probes are described in Table 1.

Tip Section. The centre hole $(C)$ with a chamfer angle of $90^{\circ}$ to the tube gives a measure of total pressure and is denoted by $P_{C}$ which is at lower left position. The side hole is chamfered at an angle of about $35^{\circ}$ to the yaw plane and is designated as $P_{S}$ and is mainly sensitive to the yaw angle variation. The bottom hole $(B)$ and top hole $(T)$ are chamfered at an angle of about $35^{\circ}$ to the pitch plane and are noted as $P_{B}$ and $P_{T}$, respectively, and are mainly sensitive to the pitch angle variation. Hence four independent probe pressures are sufficient to define the threedimensional flow. It has been already demonstrated that small chamfer gives lower sensitivity and higher operating range [22]. Tip section is made of four $0.414 \mathrm{~mm}$ diameter tubes which are inserted into four other $0.719 \mathrm{~mm}$ diameter tubes. This leaves a small gap of $0.3 \mathrm{~mm}$ between the four $0.414 \mathrm{~mm}$ diameter tubes. No attempt is made to fill this gap, as it would be very difficult to do so without distorting the extremely small diameter tubes. These small gaps also seem to have very little effect on the calibration curves. All joints of 
TABLE 1

\begin{tabular}{lcccc}
\hline \multirow{2}{*}{ Measuring tube diameter $(\mathrm{mm})$} & \multirow{2}{*}{ Holding tube diameter $(\mathrm{mm})$} & $W(\mathrm{~mm})$ & $T(\mathrm{~mm})$ & Measuring area $\left(\mathrm{mm}^{2}\right)$ \\
\hline 0.414 & 0.719 & 1.133 & 1.133 & 1.284 \\
\hline
\end{tabular}

small diameter tubes are made using Araldite. The $0.719 \mathrm{~mm}$ diameter tubes are bent at an angle of $90^{\circ}$ with a radius of curvature of about $5 \mathrm{~mm}$.

Stem Section. The stem section consists of four $1.27 \mathrm{~mm}$ diameter tubes of about $500 \mathrm{~mm}$ length which are inserted into the other ends of the $0.719 \mathrm{~mm}$ diameter tubes. To keep the four tubes in the proper plane they are brazed at different positions along its length. To maintain the position of the inner tubes fixed with respect to the stem, the outer tube of $3.175 \mathrm{~mm}$ inner diameter is also brazed with these tubes. This tube of $4 \mathrm{~mm}$ outer diameter acts as the probe holder.

\section{Calibration Tunnel, Device, Procedure, and Program}

An open jet low speed calibration tunnel facility of Thermal Turbomachines Laboratory, Department of Mechanical Engineering, IIT Madras, is used for calibration of the miniature four-hole probe as shown in Figure 3.

Calibration device is made of base plate, $c$ clamp, protractors, and pointers for measurement of pitch $(\beta)$ and yaw $(\alpha)$ angles. The twenty-channel selection box and the FC012 digital micromanometer with a range of 1-200 $\mathrm{mm}$ of water and sensitivity of $0.1 \mathrm{~mm}$ of differential air pressure are used to measure the probe pressures. The micromanometer uses the output signals from the selection box to get the velocity and pressure readings.

Calibration of the probe is carried out at a velocity of $50 \mathrm{~m} / \mathrm{s}$. The probe is mounted on the probe holder with the help of sleeve such that the pressure sensing holes of the probe are to face the flow. The assembly of the probe and probe holder is kept $100 \mathrm{~mm}$ away from the exit of the nozzle.

At first the zeroing of probe is done by setting up the pitch angle $(\beta)$ to zero degree. Initially, by changing the yaw angle $(\alpha)$, set the position of the probe such that the pressure sensed by centre hole is maximum and the yaw angle corresponding to the maximum pressure sensed by centre hole is noted down and then the probe is rotated on both positive and negative sides of the yaw angle until the pressure at the centre hole is equal to the half of the maximum pressure sensed and the corresponding yaw angles are noted down. The mean of these yaw angles is taken as zero reference yaw angle.

After fixing the zero reference position the probe is calibrated by changing $\alpha$ and $\beta$ in the range of $-40^{\circ}$ to $40^{\circ}$ with an interval of $5^{\circ}$. The calibration is done by keeping $\alpha$ constant and by varying $\beta$. For every combination of $\alpha$ and $\beta$, the probe pressures are recorded.

\section{Results and Discussion}

5.1. Sample Data. To determine the qualitative accuracy of the measurements, the measured pressure data is nondimensionalised with dynamic pressure and variation of various nondimensional probe pressures presented against yaw and pitch angles in Figure 4. Pressure measured by the central hole, $P_{C}$, is maximum at smaller yaw and pitch angles and varies more or less symmetrically about both yaw and pitch angles. Pressure measured by the side hole, $P_{S}$, seems to be mainly sensitive to yaw angle. Pressures measured by the bottom hole, $P_{B}$, and top hole, $P_{T}$, are mainly sensitive to pitch angle. The graphs show the expected trends of measured pressures without any abnormal values. Hence the data is found satisfactory and is used to determine calibration coefficients.

5.2. Calibration Coefficients. The calibration coefficients are defined as follows:

$$
\begin{gathered}
P_{\mathrm{BAR}}=\frac{\left(P_{S}+P_{B}+P_{T}\right)}{3}, \\
D=P_{C}-P_{\mathrm{BAR}}, \\
C_{\mathrm{PYAW}}=\frac{\left(P_{\mathrm{C}}-P_{S}\right)}{D}, \\
C_{\mathrm{PPITCH}}=\frac{\left(P_{T}-P_{B}\right)}{D}, \\
C_{\mathrm{PTOTAL}}=\frac{\left(P_{\mathrm{C}}-P_{\mathrm{O}}\right)}{\mathrm{Q}}, \\
C_{\mathrm{PSTATIC}}=\frac{\left(P_{\mathrm{BAR}}-P_{\mathrm{ST}}\right)}{\mathrm{Q}}, \\
=\frac{\left(P_{\mathrm{O}}-P_{\mathrm{ST}}=P_{\mathrm{O}} \quad\left(\text { as } P_{\mathrm{ST}}=0\right)\right.}{P_{\mathrm{O}}} \\
=\frac{P_{\mathrm{BAR}}}{P_{\mathrm{O}}} .
\end{gathered}
$$

Calibration Curves. The following calibration curves are presented in Figure 4. For the sake of clarity, calibration curves are shown at $10^{\circ}$ intervals only.

(1) $C_{\text {PYAW }}$ versus $C_{\text {PPITCH }}$ for different pitch and yaw angles.

(2) $C_{\text {PTOTAl }}$ and $C_{\text {PSTATIC }}$ contours with $C_{\text {PPITCH }}$ and $C_{\text {PYAW }}$ along the axis.

In Figure 4, calibration coefficients at $\alpha=-40^{\circ}$ and $\beta=-40^{\circ}$ are not presented. The value of probe dynamic pressure, $D$ is very small and the magnitude of the calibration coefficients is very large.

For an ideal five-hole probe, $C_{\text {PYAW }}$ versus $C_{\text {PPITCH }}$ calibration curves at constant yaw angles will be horizontal 


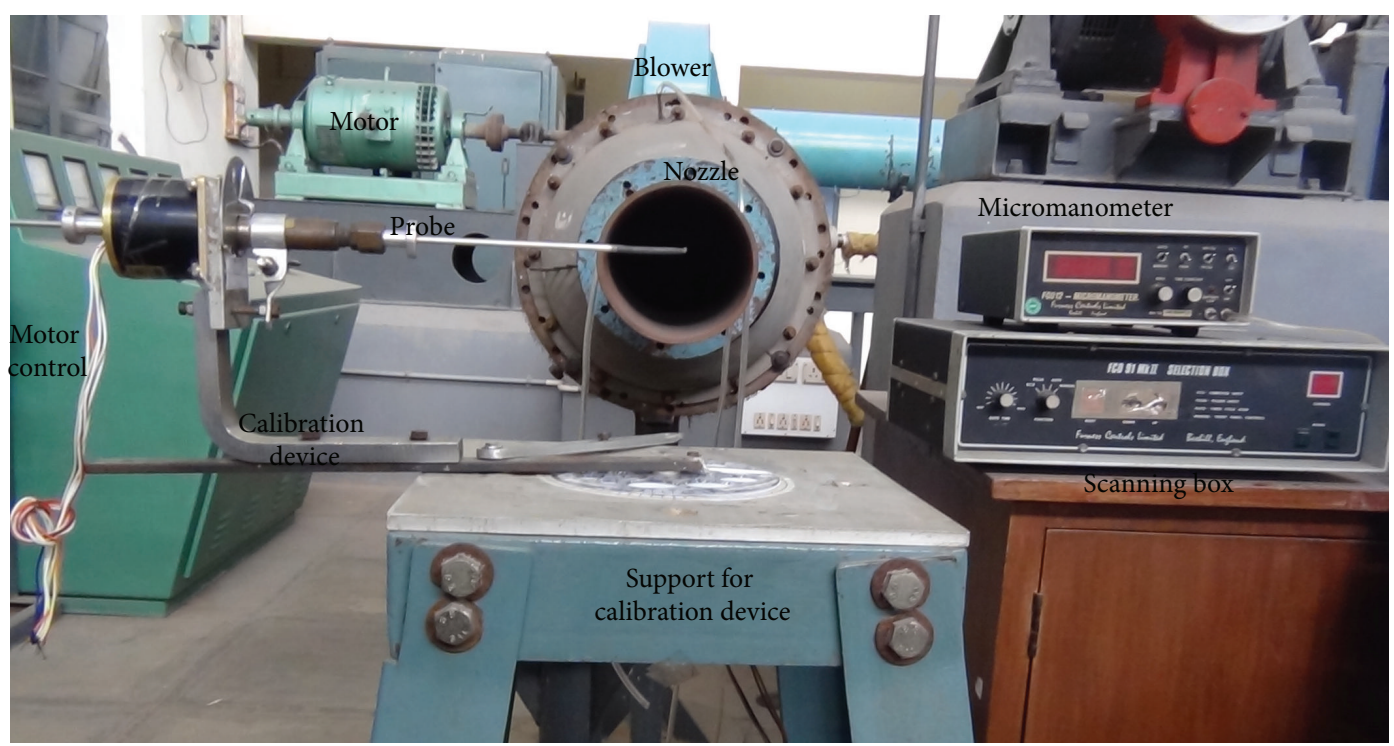

FIGURE 3: Calibration tunnel, calibration device, probe, and instrumentation.
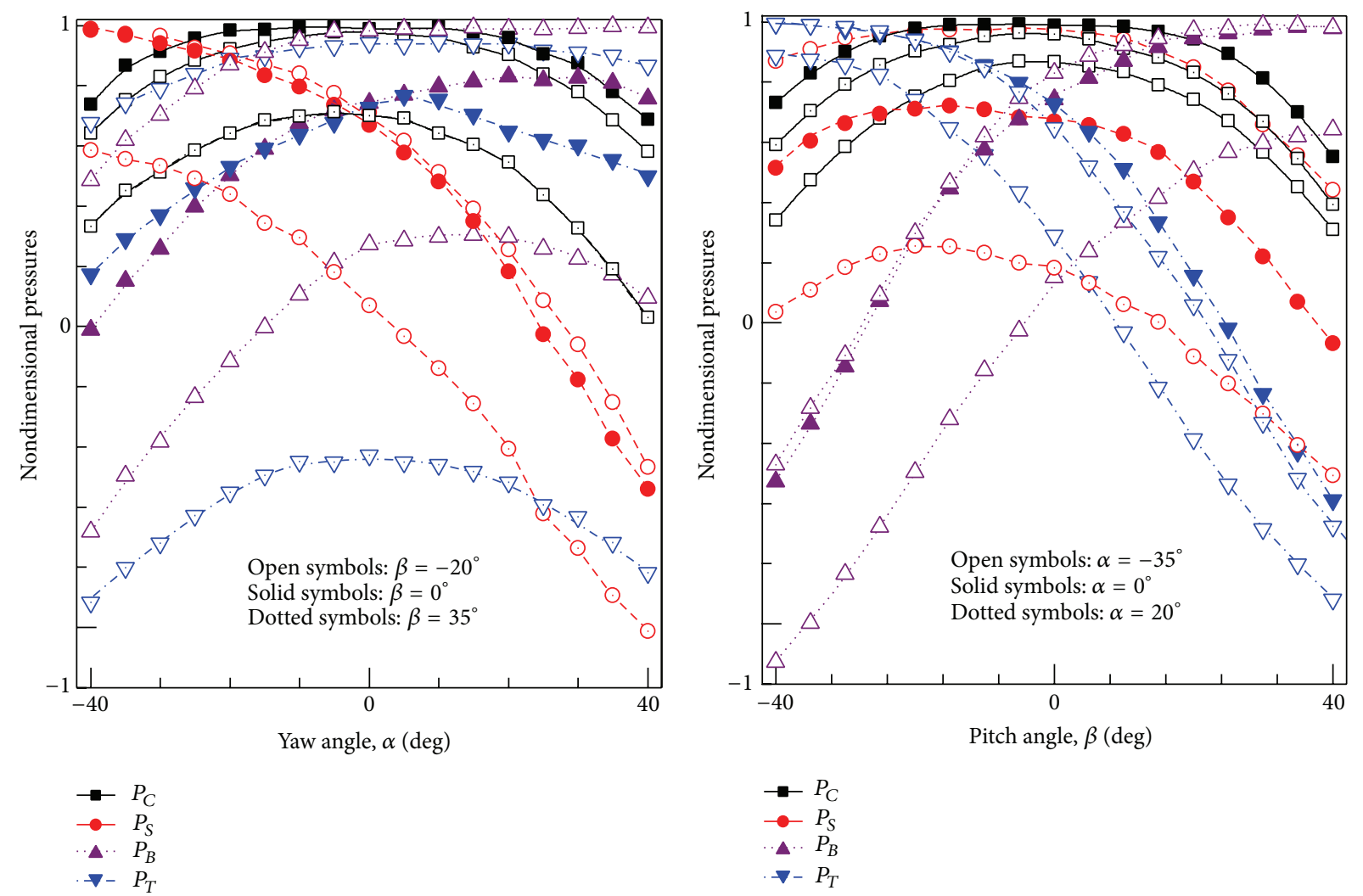

Figure 4: Sample data.

and symmetrical about zero yaw angle. $C_{\text {PYAW }}$ versus $C_{\text {PPITCH }}$ calibration curves at constant pitch angles will be vertical and symmetrical about zero pitch angle. Because of nonlinearity in the behaviour of static pressure, these calibration curves will be curved. For a four-hole probe, the calibration curves will be asymmetric about both yaw and pitch angles at zero value. In $C_{\text {PYAW }}$ versus $C_{\text {PPITCH }}$ curves for $\beta=20^{\circ}, 30^{\circ}$, and $40^{\circ}, C_{\text {PPITCH }}$ is minimum at $\alpha=0^{\circ}$ and increases nonlinearly on both sides of the zero yaw angle. For $\beta=-20^{\circ},-10^{\circ}, 0^{\circ}$, and $10^{\circ}, C_{\text {PPITCH }}$ is found to vary nonlinearly for different values of yaw angles. For $\beta=-30^{\circ}$ and $-40^{\circ}, C_{\text {PPITCH }}$ is maximum at $\alpha=0^{\circ}$ and decreases nonlinearly on both sides 


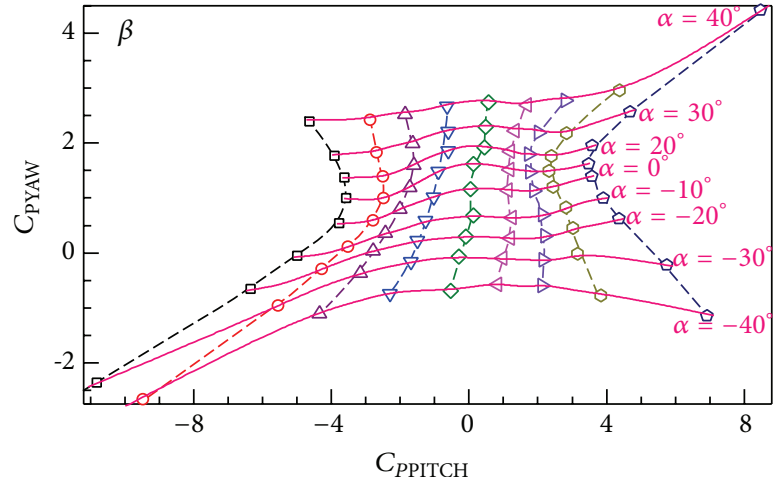

$$
\begin{array}{ll}
-\square--40^{\circ} & -\triangleleft-10^{\circ} \\
-\odot--30^{\circ} & -\triangleright-20^{\circ} \\
-\triangle--20^{\circ} & -\diamond-30^{\circ} \\
-\nabla--10^{\circ} & -\diamond-40^{\circ} \\
-\diamond-0^{\circ} &
\end{array}
$$

(a)

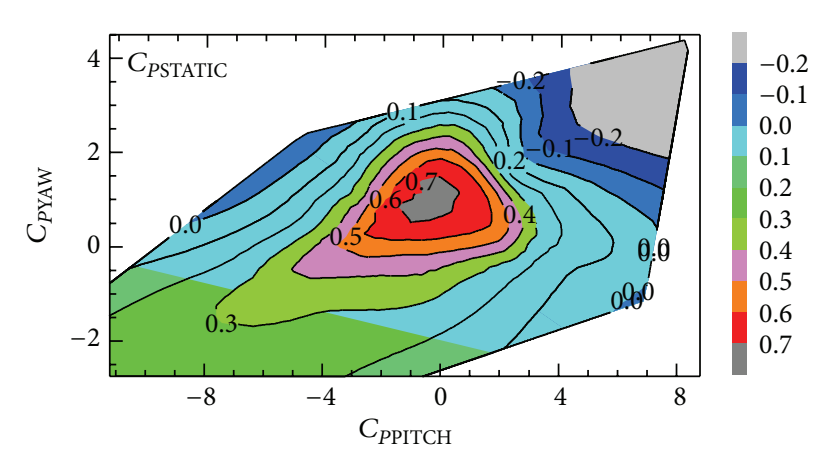

(c)

FIGURE 5: Calibration curves of miniature four-hole probe.

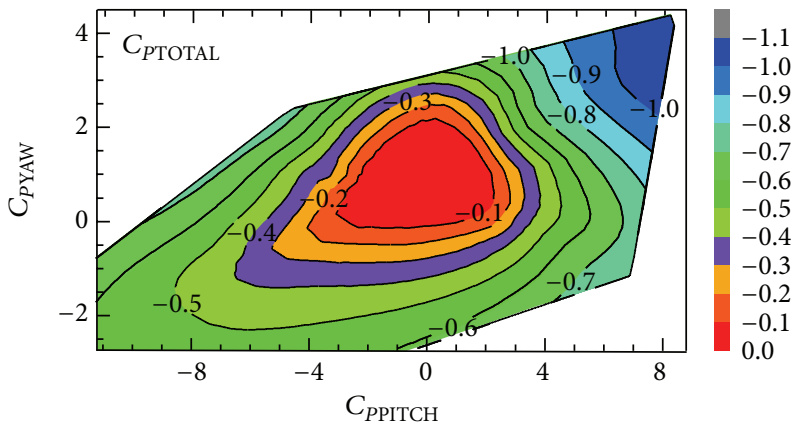

(b) of the yaw angles. At $\alpha=0^{\circ}, C_{\text {PYAW }}$ is almost a straight line for various pitch angles and as $\alpha$ increases on both sides $C_{\text {PYAW }}$ varies nonlinearly for different values of pitch angles as shown in Figure 4. From Figure 4, it is evident that the pitch coefficient, $C_{\text {PPITCH }}$, has larger sensitivity with pitch angle compared to $C_{\text {PYAW }}$ sensitivity with yaw angle. This is expected as $C_{\text {PPITCH }}$ depends on two pitch holes compared to $C_{\text {PYAW }}$ which depends on one yaw hole only.

In the $C_{\text {PTOTAL }}$ and $C_{\text {PSTATIC }}$ contours (Figure 5), the minimum $C_{\text {PTOTAL }}$ contour is 0 at zero yaw and pitch angles. By definition of the $C_{\text {PTOTAL }}$, as the pressure sensed by the centre hole is always less than the total pressure, $C_{\text {PTOTAL }}$ can be either zero or negative. At pitch and yaw angles away from zero values, $C_{\text {PTOTAL }}$ becomes more negative.

The minimum $C_{\text {PSTATIC }}$ contour is 0.7 at small yaw and pitch angles because it depends on $P_{\mathrm{BAR}}$ and $P_{\mathrm{BAR}}$ is average pressure sensed by side, bottom, and top holes. Hence the value of $C_{\text {PSTATIC }}$ decreases at higher yaw and pitch angles because $P_{\mathrm{BAR}}$ decreases rapidly at higher yaw and pitch angles.

5.3. Sensitivity Analysis of Calibration Coefficients. To define the accuracy of the measurements, sensitivity analysis of calibration data is carried out. The sensitivity coefficients are defined as a function of yaw or pitch angle while keeping pitch or yaw angle constant. The sensitivity coefficients are shown in Figure 6.

Sensitivity coefficients are defined as

$$
\Delta C_{P_{i}}=\frac{\left(C_{P_{(i+1)}}-C_{P_{(i-1)}}\right)}{(\operatorname{Angle}(i+1)-\operatorname{Angle}(i-1))},
$$

where $C_{P}$ is the one of the four calibration coefficients, namely $C_{\text {PYAW }}, C_{\text {PPITCH }}, C_{\text {PTOTAL }}$, and $C_{\text {PSTATIC }}$, and $i$ is the yaw or pitch angle where the calibration data is taken.

The probe pressures change rapidly at large yaw and pitch angles. Therefore calibration coefficients at large values of yaw and pitch angles have higher sensitivity. Although higher sensitivity implies more accurate measurements but operating range of the probe will be less. It is to be kept in mind that small error in pressures results in large errors in calibration coefficients and their sensitivity. At low values of yaw and pitch angles, sensitivity coefficients are low. The probe hole chamfered angles are small, about $35^{\circ}$. It is already demonstrated that small chamfer angles result in 

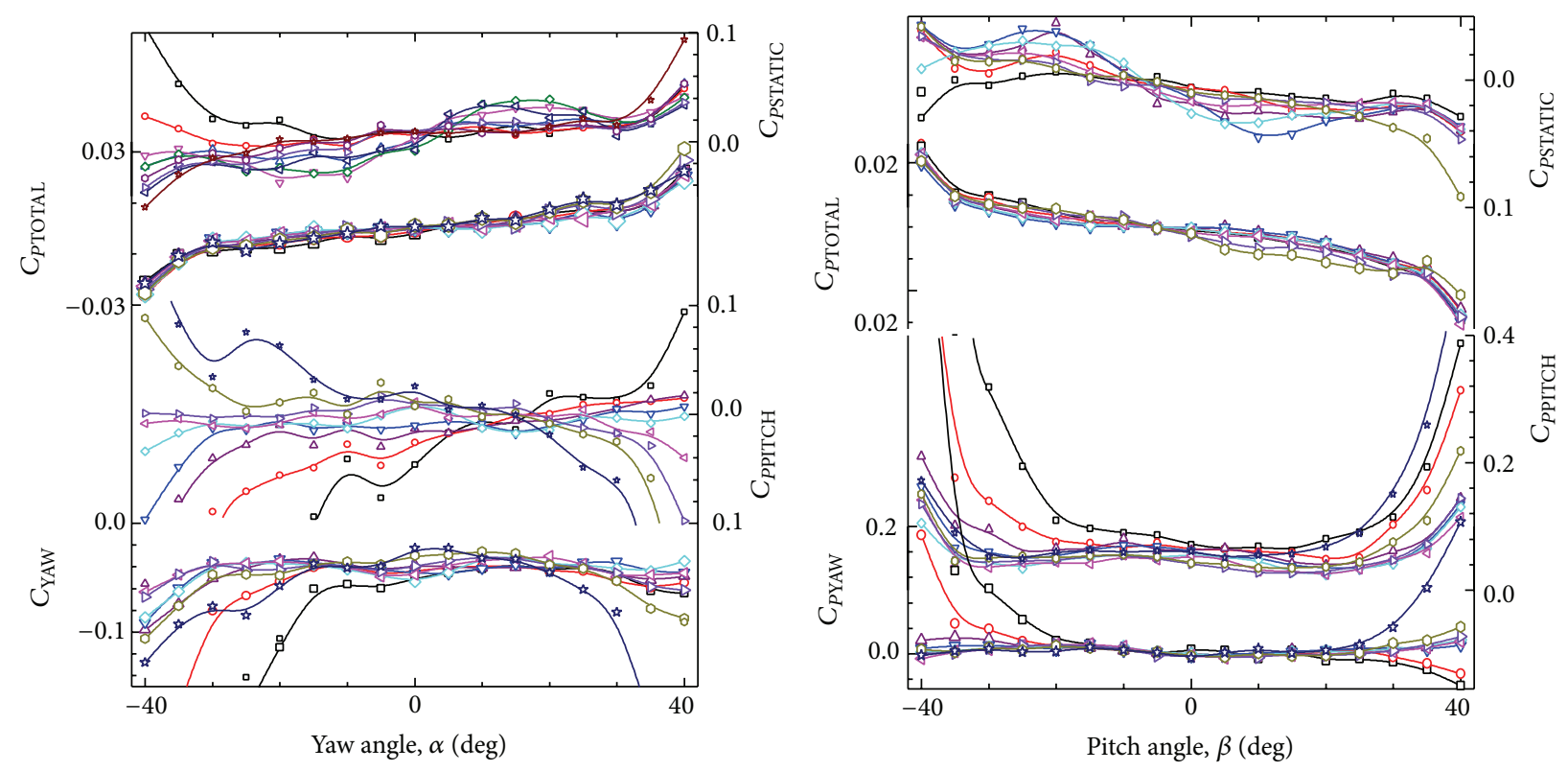

$$
\begin{array}{ll}
-\square- & -40^{\circ} \\
-\bigcirc- & -30^{\circ} \\
-- & -20^{\circ} \\
-\nabla- & -10^{\circ} \\
\checkmark- & 0^{\circ}
\end{array}
$$$$
\checkmark 10^{\circ}
$$$$
\rightarrow 20^{\circ}
$$$$
-10-30^{\circ}
$$$$
\text { is } 40^{\circ}
$$

$$
\begin{aligned}
& -\square-40^{\circ} \\
& -\bigcirc-30^{\circ} \\
& -\square-20^{\circ} \\
& -\nabla-10^{\circ}
\end{aligned}
$$$$
\begin{aligned}
& \rightarrow-20^{\circ} \\
& \rightarrow-30^{\circ}
\end{aligned}
$$$$
\rightarrow-40^{\circ}
$$

FIGURE 6: Sensitivity curves of calibration coefficients.

lower sensitivity and large chamfer angles result in higher sensitivity [22].

5.4. Validation of Calibration Data. Sitaram and Kumar [23] developed a look up table method to determine the four unknown quantities, namely, yaw and pitch angles and static and total pressure coefficients from the calculated yaw and pitch coefficients of a five-hole probe. The yaw and pitch coefficients are calculated from the measured pressure data. The same method is utilised for determining the flow quantities from the present four-hole probe measurements. No additional data is taken for interpolation during the calibration of the probe. However the calibration data at intervals of $10^{\circ}$ rather than $5^{\circ}$ are used. All the calibration data are used as measured data. A calibration interval of $10^{\circ}$ is large. Sumner [24] recommended that this is the largest calibration interval that can be used with a seven-hole probe.

The interpolated values are compared with those obtained during calibration. Histograms of errors in yaw and pitch angles are presented in Figure 7 . The errors at the extremes of the calibration range, that is, $\pm 40^{\circ}$ of yaw and pitch angles, are omitted from these graphs. Most of the errors in yaw and pitch angles are within $\pm 1^{\circ}$ and most of the errors in total, static, and dynamic pressures are within -0.005 to $0.01 \%$ of the dynamic head.

The maximum absolute, average, and rms values of errors in yaw and pitch angles and total, static, and dynamic pressures are also presented in Table 2.
Except for yaw angle and dynamic pressure, the errors are very small. The large values of error occur near the extreme range of calibration. The errors are due to the data reduction program only. All other measurement errors such as instrumentation errors, errors due calibration (zero angle settings, pitch and yaw angle measurements during calibration, etc.), are not included. The calibration data is given in 10 deg. interval. The calibration data in 5 deg. interval (excluding the data at $10 \mathrm{deg}$. interval) are given as measured data. The errors are almost negligible when both calibration data and measured data are given in $5 \mathrm{deg}$. interval. The errors presented by Lee and Jun [7], who used a calibration interval of $5^{\circ}$ in their data reduction program, have similar magnitude.

5.5. Comparison of Measurements in a Calibration Duct. The four-hole probe along with a conventional truncated conical head with perpendicular holes ( $3 \mathrm{~mm}$ dia. head) and an extremely small Pitot tube is used to measure the flow across the calibration section of the calibration tunnel available at Thermal Turbomachines Laboratory, Department of Mechanical engineering, IIT Madras $(0.5 \mathrm{~mm}$ tip dia. of $5 \mathrm{~mm}$ length; this tube is extended to $0.8 \mathrm{~mm}$ dia. tube, followed by $1.27 \mathrm{~mm}$ dia. tube to reduce the response time). A schematic of the calibration tunnel is shown in Figure 8.

The circumferentially averaged wall static pressure of the settling chamber is equal to the total pressure in the calibration section. The static pressure at the measurement station is measured by means of four circumferentially 

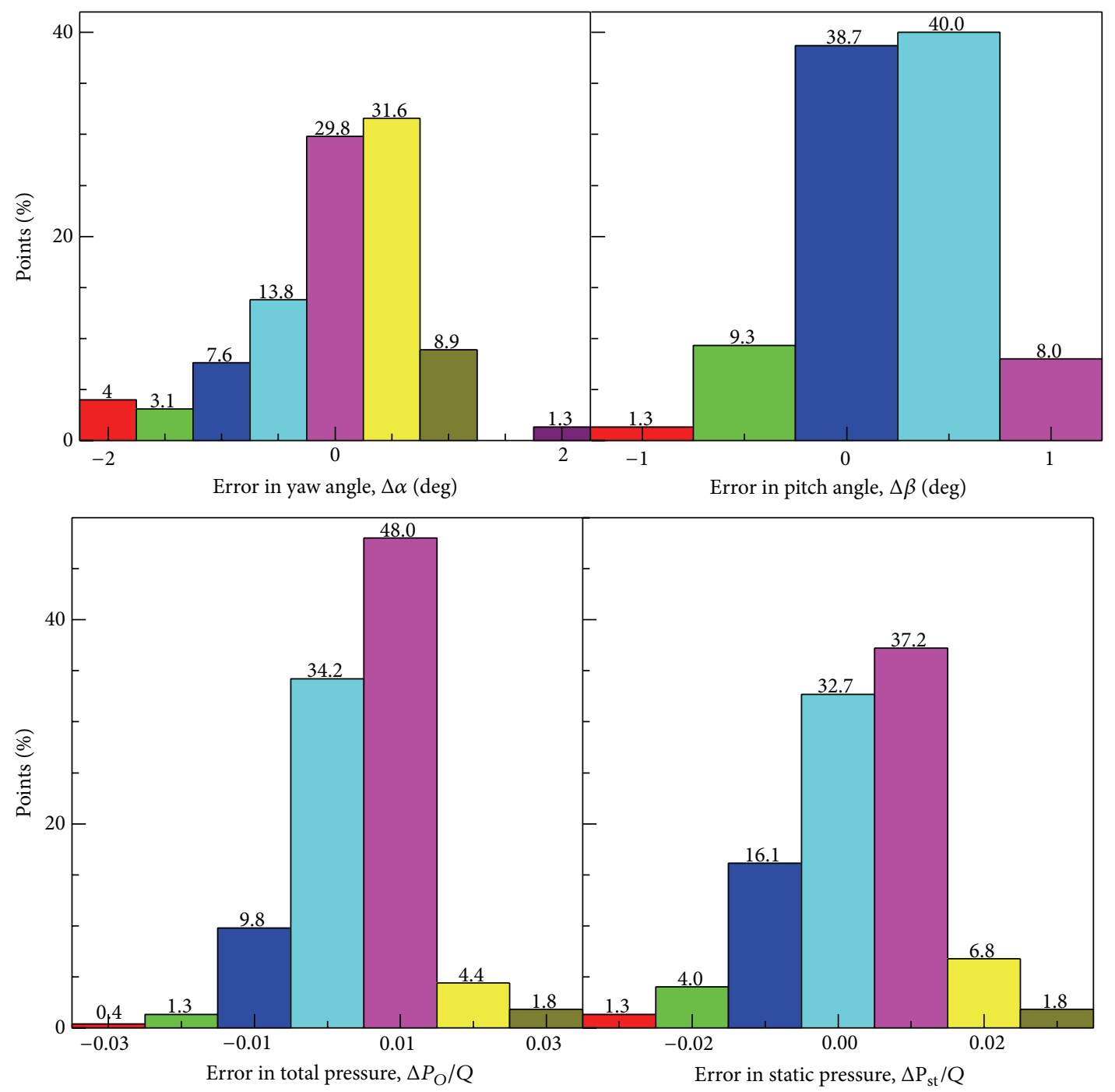

FIGURE 7: Histograms of interpolated errors in percentages.

TABLE 2

\begin{tabular}{lcccc}
\hline Parameter & Maximum error & Minimum error & Average value & RMS value \\
\hline$\Delta \alpha$ & $1.7^{\circ}$ & $-2.4^{\circ}$ & $-0.26^{\circ}$ & $0.80^{\circ}$ \\
$\Delta \beta$ & $1.3^{\circ}$ & $-1.1^{\circ}$ & $0.01^{\circ}$ & $0.42^{\circ}$ \\
$\Delta P_{\mathrm{O}} / Q$ & 0.034 & -0.031 & -0.001 & 0.008 \\
$\Delta P_{\mathrm{ST}} / Q$ & 0.026 & -0.034 & -0.002 & 0.012 \\
$\Delta Q / Q$ & 0.029 & -0.049 & 0.003 & 0.011 \\
\hline
\end{tabular}

averaged wall static pressure taps. The probes are traversed from the centre of the calibration section to the end of the opposite wall. A manual traversing mechanism with $1 \mathrm{~mm}$ measurement resolution along the radial direction and $1^{\circ}$ measurement resolution in the yaw plane is used to traverse the probes. The three probes are nulled at the centre of the calibration section and traversed in large intervals $(10 \mathrm{~mm})$ near the centre. As the probes approach the opposite wall, the intervals are reduced to $5 \mathrm{~mm}, 2 \mathrm{~mm}$, and $1 \mathrm{~mm}$. The results of these measurements are presented in Figure 9.
From Figure 9, it is evident that the nondimensional total pressure and velocity measured by all the three probes are in good agreement at the centre and away from the centre up to a distance of 0.2 times radius from the calibration section wall. For the Pitot tube, wall static pressure is used to calculate velocity. The flow is uniform in this region. The thickness of the boundary layer is about 0.2 times the radius of the calibration section. The nondimensional static pressures measured by the four-hole and five-hole probes are in good agreement with the nondimensional static pressure 


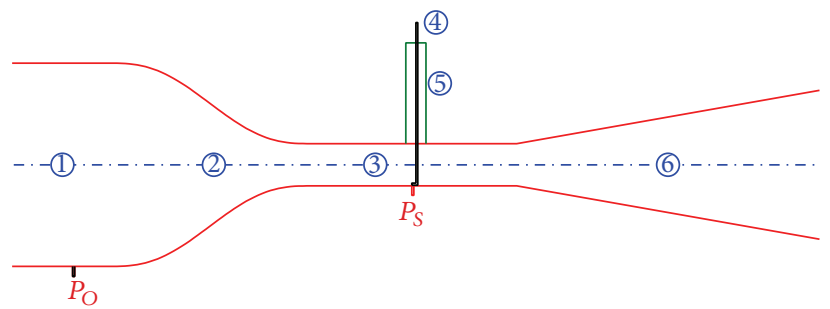

(1) Settling chamber

(2) Contraction

(3) Calibration section

(4) Probe
(5) Traverse mechanism

(6) Diverging duct

$P_{O}$ settling chamber static pressure

$P_{S}$ calibration section static pressure

FIGURE 8: Schematic of calibration tunnel.

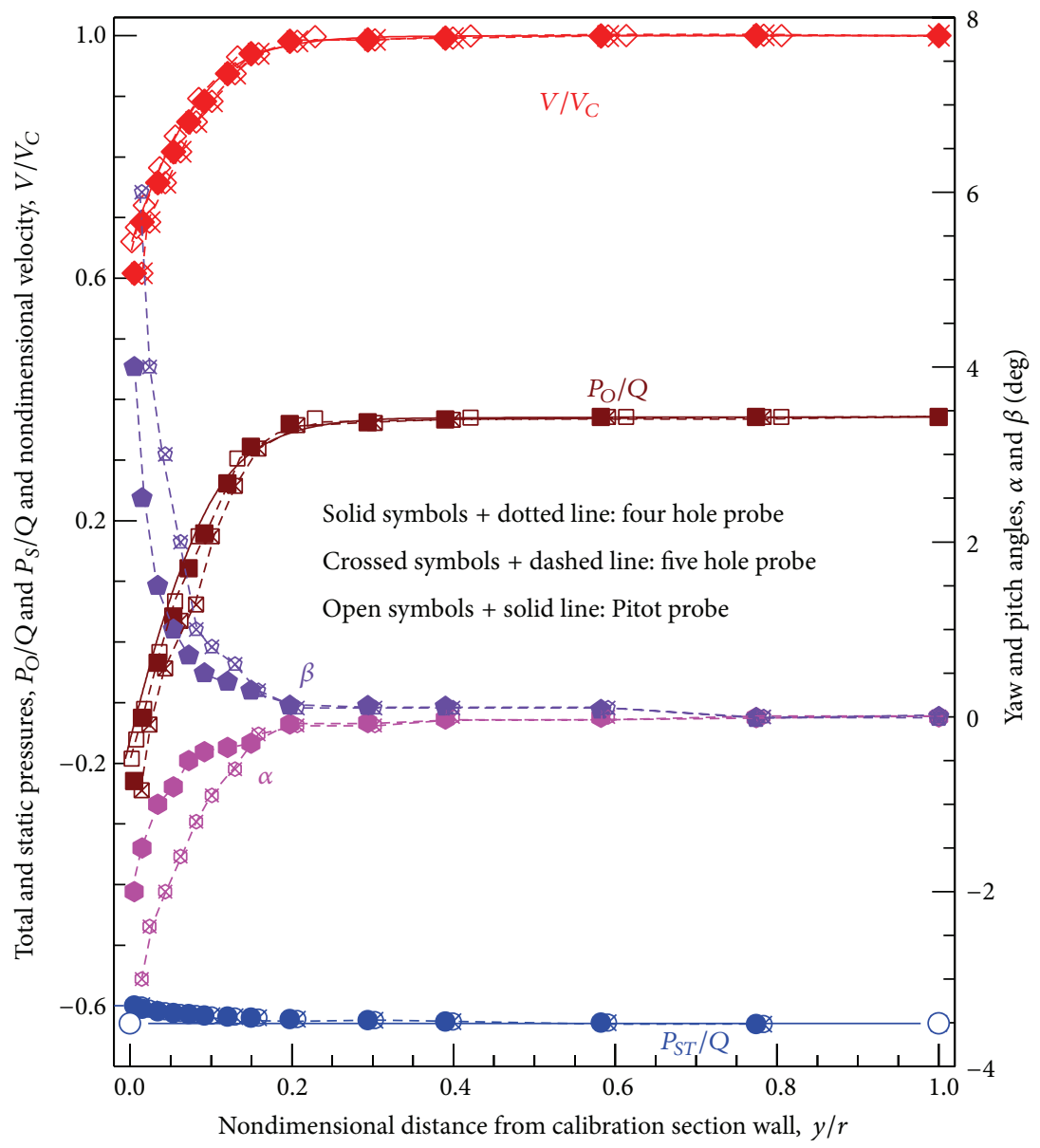

$\begin{array}{ll}- & P_{\mathrm{O}} / \mathrm{Q} \\ - & \alpha \\ - & P_{S T} / \mathrm{Q}\end{array}$

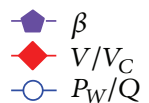

FIGURE 9: Comparison of probe measurements in the calibration section of the calibration tunnel. 
measured by the wall static pressure taps. Only very near the wall of the calibration section, the static pressures measured by the probes are slightly higher than that measured by the wall static pressure taps.

Near the wall, pressure probes suffer from two major sources of errors, namely, pressure or velocity gradients and surface proximity errors. In addition, the turbulence intensity increases as the probes approach the walls. Recent investigations [25] have shown that the turbulence intensity affects the calibration characteristics of multihole probes and suggested that the multihole probes should be calibrated at the same turbulence intensity of the flow to be measured. Sitaram et al. [26] have discussed these and other sources of errors in the application of five-hole probes and they gave estimates of their magnitudes. Near the wall, the flow is modified due to the presence of the probe and the probe gives erroneous results. These erroneous results are usually confined to a distance of about twice the diameter of the probe. All the parameters that are measured, namely, total and static pressures, flow angles, and velocity and its three components, are affected due to the surface proximity. However, usually static pressure and pitch angle show larger errors. As the tip diameter of the Pitot tube is only $0.5 \mathrm{~mm}$, it can measure total pressure accurately $1 \mathrm{~mm}$ away from the wall. The nominal sizes of the four-hole and five-hole probes are $1.1 \mathrm{~mm}$ and $3 \mathrm{~mm}$, respectively, and the errors measured by these probes extend up to distances of about 2 and $6 \mathrm{~mm}$, respectively. This can be clearly demonstrated by examination of the radial distribution of yaw and pitch angles.

The radial distribution of yaw and pitch angles measured by the four-hole and five-hole probes is also presented in Figure 9. At the centre of the calibration section, both yaw and pitch angles are zero as the flow is one-dimensional and aligned with the axis of the calibration section. These angles are close to zero up to very close to the wall. The small discrepancies can be attributed to the interpolation errors. As expected, the angles measured by the four-hole probe are nonzero very close to the wall $(y / r \leq 0.02)$. For the fivehole probe, the angles measured are nonzero and are up to $y / r=0.06$. The maximum values of yaw angles are -2 and -3 , for the four-hole and five-hole probes, respectively. The maximum values of pitch angles are 4 and 6, respectively, for the four-hole and five-hole probes.

\section{Conclusions}

A miniature four-hole probe with minimum spatial error is designed and fabricated. The probe can be used to analyze three-dimensional flows with large pressure and velocity gradients in all directions as in cases of tip clearance vortex, flows in corner of blades, end wall flows, and other complex flows that occur in turbomachinery. The probe is calibrated in the range of $-40^{\circ}$ to $40^{\circ}$ in both yaw and pitch planes with an interval of $5^{\circ}$. Calibration coefficients are defined, determined, and plotted. Sensitivity analysis of the calibration data is also performed. A lookup table method is used to interpolate the four unknown quantities, namely, total and static pressures and flow angles. The maximum absolute errors in yaw and pitch angles are 2.4 and $1.3 \mathrm{deg}$. respectively. The maximum absolute errors in total, static and dynamic pressures are $3.4,3.9$ and $4.9 \%$ of the dynamic pressures respectively. Measurements made with this probe, a conventional five-hole probe and a miniature Pitot probe across a calibration section, demonstrated that the errors due to gradient and surface proximity for this probe are considerably reduced compared to the five-hole probe. Hence, this probe is more suitable to measure three-dimensional flows with large pressure and velocity gradients as in cases of tip clearance vortex, flows in corner of blades, end wall flows, and other complex flows that occur in turbomachinery.

\section{Nomenclature}

\begin{tabular}{|c|c|}
\hline$C_{\text {PPITCH }}:$ & Pitch coefficient \\
\hline$C_{\text {PSTATIC }}:$ & Static pressure coefficient \\
\hline$C_{\text {PTOTAL }}:$ & Total pressure coefficient \\
\hline$C_{\text {PYAW }}:$ & Yaw coefficient \\
\hline$D:$ & Probe dynamic pressure $(\mathrm{Pa})$ \\
\hline$P_{B}:$ & Pressure sensed by bottom hole $(\mathrm{Pa})$ \\
\hline$P_{C}:$ & Pressure sensed by centre hole $(\mathrm{Pa})$ \\
\hline$P_{\mathrm{BAR}}:$ & $\begin{array}{l}\text { Average pressure sensed by chamfered } \\
\text { holes (defined in text) }\end{array}$ \\
\hline$P_{O}:$ & Total Pressure $(\mathrm{Pa})$ \\
\hline$P_{S}:$ & Pressure sensed by side hole $(\mathrm{Pa})$ \\
\hline$P_{\mathrm{ST}}:$ & Static Pressure $(\mathrm{Pa})$ \\
\hline$P_{T}:$ & Pressure sensed by top hole $(\mathrm{Pa})$ \\
\hline$P_{w}:$ & $\begin{array}{l}\text { Static pressure measured by wall taps of } \\
\text { the calibration section }(\mathrm{Pa})\end{array}$ \\
\hline Q: & Dynamic pressure $(\mathrm{Pa})$ \\
\hline$r:$ & Radius of calibration section (m) \\
\hline$V:$ & Velocity in the calibration section $(\mathrm{m} / \mathrm{s})$ \\
\hline$V_{C}:$ & $\begin{array}{l}\text { Velocity at the centre of the calibration } \\
\text { section }(\mathrm{m} / \mathrm{s})\end{array}$ \\
\hline$y:$ & $\begin{array}{l}\text { Distance from the wall of the calibration } \\
\text { section }(\mathrm{m})\end{array}$ \\
\hline$\alpha:$ & Yaw angle (deg.) \\
\hline$\beta:$ & Pitch angle (deg.) \\
\hline$\Delta \alpha, \Delta \beta$ & $\begin{array}{l}\text { Errors in interpolated values of yaw and } \\
\text { pitch angles (deg.) }\end{array}$ \\
\hline$\Delta P_{\mathrm{O}}, \Delta P_{\mathrm{ST}}:$ & $\begin{array}{l}\text { Errors in interpolated values of total and } \\
\text { static pressures }(\mathrm{Pa})\end{array}$ \\
\hline$C:$ & $\begin{array}{l}\text { Value at the centre of the calibration } \\
\text { section. }\end{array}$ \\
\hline
\end{tabular}

\section{Conflict of Interests}

The authors declare that there is no conflict of interests regarding the publication of this paper.

\section{Acknowledgments}

The authors would like to thank Mr. M. Veeraraghavan and Mr. N. Giri of Thermal Turbomachines Laboratory, Department of Mechanical Engineering, IIT Madras, for fabrication of the miniature four-hole probe. The authors 
would like to thank Mr. R. Bharath Viswanath, Project Associate, Thermal Turbomachines Laboratory, Department of Mechanical Engineering, IIT Madras, for his help in running lookup table method program. The authors would like to thank the reviewers for their suggestions, which improved the quality of the paper substantially.

\section{References}

[1] A. L. Treaster and A. M. Yocum, "The calibration and application of five-hole probes," nstrumentation Society of America Transactions, vol. 18, no. 3, pp. 23-34, 1979.

[2] A. J. Pisasale and N. A. Ahmed, "A novel method for extending the calibration range of five-hole probe for highly threedimensional flows," Flow Measurement and Instrumentation, vol. 13, no. 1-2, pp. 23-30, 2002.

[3] A. Pissasale and N. A. Ahmed, "Theoretical calibration for highly three-dimensional low-speed flows of a five-hole probe," Measurement Science and Technology, vol. 13, no. 7, pp. 11001107, 2002.

[4] A. Pissasale and N. A. Ahmed, "Development of a functional relationship between port pressures and flow properties for the calibration and application of multihole probes to highly threedimensional flows," Experiments in Fluids, vol. 36, no. 3, pp. 422-436, 2004.

[5] T. Yasa and G. Paniagua, "Robust procedure for multi-hole probe data processing," Flow Measurement and Instrumentation, vol. 26, pp. 46-54, 2012.

[6] R. G. Dominy and H. P. Hodson, "Investigation of factors influencing the calibration of five-hole probes for three-dimensional flow measurements," Journal of Turbomachinery, vol. 115, no. 3, pp. 513-519, 1993.

[7] S. W. Lee and S. B. Jun, "Reynolds number effects on the non-nulling calibration of a cone-type five-hole probe for turbomachinery applications," Journal of Mechanical Science and Technology, vol. 19, no. 8, pp. 1632-1648, 2005.

[8] D. Telionis, Y. Yang, and O. Rediniotis, "Recent developments in multi-hole probe technology," in Proceedings of 20th International Congress of Mechanical Engineering (COBEM '09), Gramado, Brazil, November 2009.

[9] S. J. Lien and N. A. Ahmed, "An examination of suitability of multi-hole pressure probe technique for skin friction measurement in turbulent flow," Flow Measurement and Instrumentation, vol. 22, no. 3, pp. 153-164, 2011.

[10] P. M. Ligrani, B. A. Singer, and L. R. Baun, "Miniature fivehole pressure probe for measurement of three mean velocity components in low-speed flows," Journal of Physics E: Scientific Instruments, vol. 22, no. 10, pp. 868-876, 1989.

[11] V. Chernoray and J. Hjärne, "Improving the accuracy of multihole probe measurements in velocity gradients," in Proceedings of the ASME Turbo Expo, pp. 125-134, Berlin, Germany, June 2008.

[12] J. Town, A. Akturk, and C. Camci, "Total pressure correction of a sub-miniature five-hole probe in areas of pressure gradients," in Proceedings of the ASME Turbo Expo 2012: Turbine Technical Conference and Exposition (GT '12), pp. 855-861, Copenhagen, Denmark, June 2012.

[13] H. T. Hoenen, R. Kunte, P. Waniczek, and P. Jeschke, "Measuring failures and correction methods for pneumatic multihole probes," in Proceedings of the ASME Turbo Expo : Turbine Technical Conference and Exposition (GT'12), pp. 721-729, Copenhagen, Denmark, June 2012.
[14] J. R. Erwin, "Experimental techniques: instrumentation," in High Speed Aerodynamics and Jet Propulsion, vol. 5 of Aerodynamics of Turbines and Compressors, p. 173, 1964.

[15] N. K. Maheshwari, D. Saha, R. K. Sinha, and V. Venkat Raj, Velocity Measurement in the Three Dimensional Flow Fluid in a Nuclear Reactor Calandria Using Four Hole Probe, Reactor Engineering Division, Bhabha Atomic Research Centre, Mumbai, India, 1998.

[16] AC-flow., http://ac-flow.com/upload/files/4-hole\%20wedge\%20 type\%20probe.pdf.

[17] A. Heneka, "Instantaneous three dimensional flow measurements with a four hole wedge probe," in Proceedings of the 7th Symposium on Measuring Techniques for Transonic and Supersonic Flow in Cascades and Turbomachines, Aachen, Germany, 1983.

[18] R. W. Ainsworth, J. L. Allen, and J. J. M. Batt, “The development of fast response aerodynamic probes for flow measurements in turbomachinery," ASME Journal of Turbomachinery, vol. 117, no. 4, pp. 625-634, 1995.

[19] J. P. Schlienger, Evolution of unsteady secondary flows in a multistage shrouded axial turbine [dissertation], ETH, Zürich, Switzerland, 2003, ETH no. 15230.

[20] I. C. Shepherd, "A four hole pressure probe for fluid flow measurements in three dimensions," Journal of Fluids Engineering, vol. 103, no. 4, pp. 590-594, 1981.

[21] N. Sitaram and A. L. Treaster, "A simplified method of using four-hole probes to measure three dimensional flow fields," Journal of Fluids Engineering, Transactions of the ASME, vol. 107, no. 1, pp. 31-35, 1985.

[22] N. Sitaram and K. Srikanth, "Effect of chamfer angle on the calibration curves of five hole probes," in Proceedings of the 39th National Conference on Fluid Mechanics and Fluid Power, Paper No. FMFP2012-240, p. 10, SVNIT, Surat, India, December 2012.

[23] N. Sitaram and S. Kumar, "Look up table method for five hole probe data reduction," in Proceedings of the 38th National Conference on Fluid Mechanics and Fluid Power, vol. 8 of Paper No. EM05, MANIT, Bhopal, India, December 2011.

[24] D. Sumner, "A comparison of data-reduction methods for a seven-hole probe," Journal of Fluids Engineering, vol. 124, no. 2, pp. 523-527, 2002.

[25] C. Crowley, I. I. Shinder, and M. R. Moldover, "The effect of turbulence on a multi-hole Pitot calibration," Flow Measurement and Instrumentation, vol. 33, pp. 106-109, 2013.

[26] N. Sitaram, B. Lakshminarayana, and A. Ravindranath, "Conventional probes for the relative flow measurement in a turbomachinery rotor blade passage," ASME Journal of Engineering for Gas Turbines and Power, vol. 103, no. 2, pp. 406-414, 1981. 

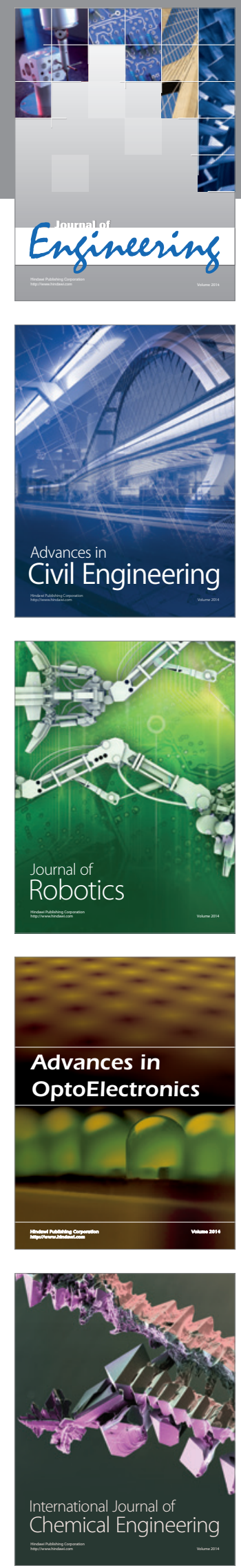

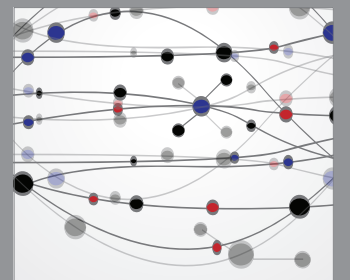

The Scientific World Journal
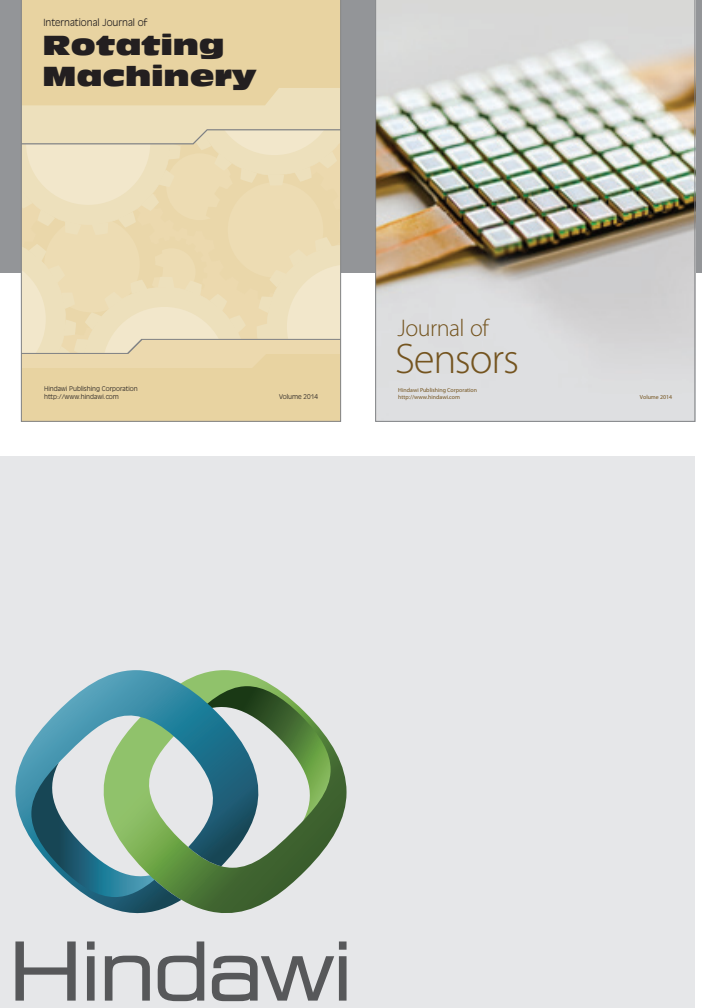

Submit your manuscripts at http://www.hindawi.com
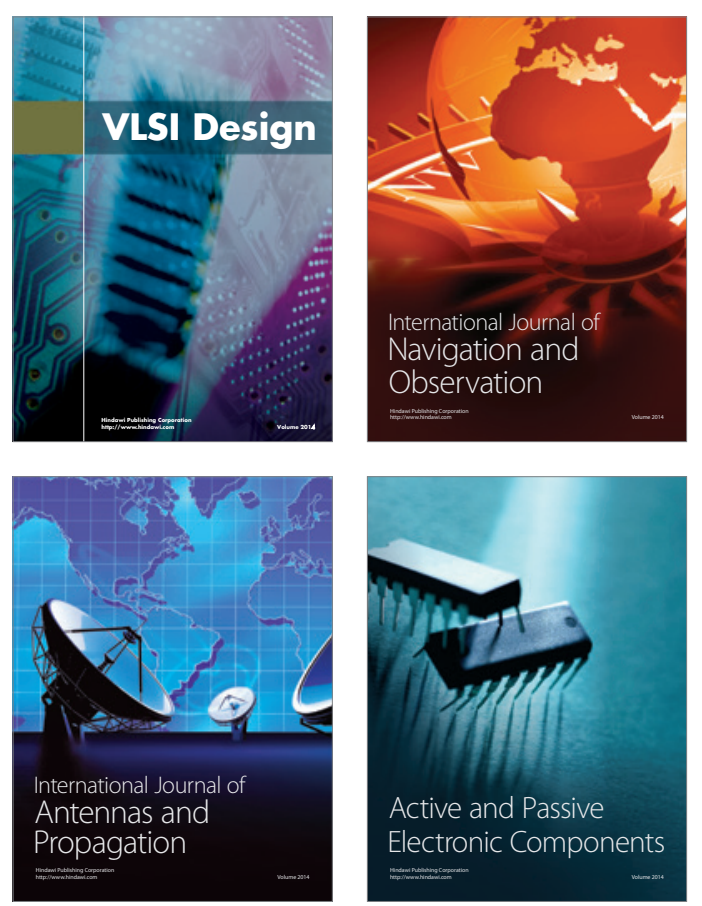
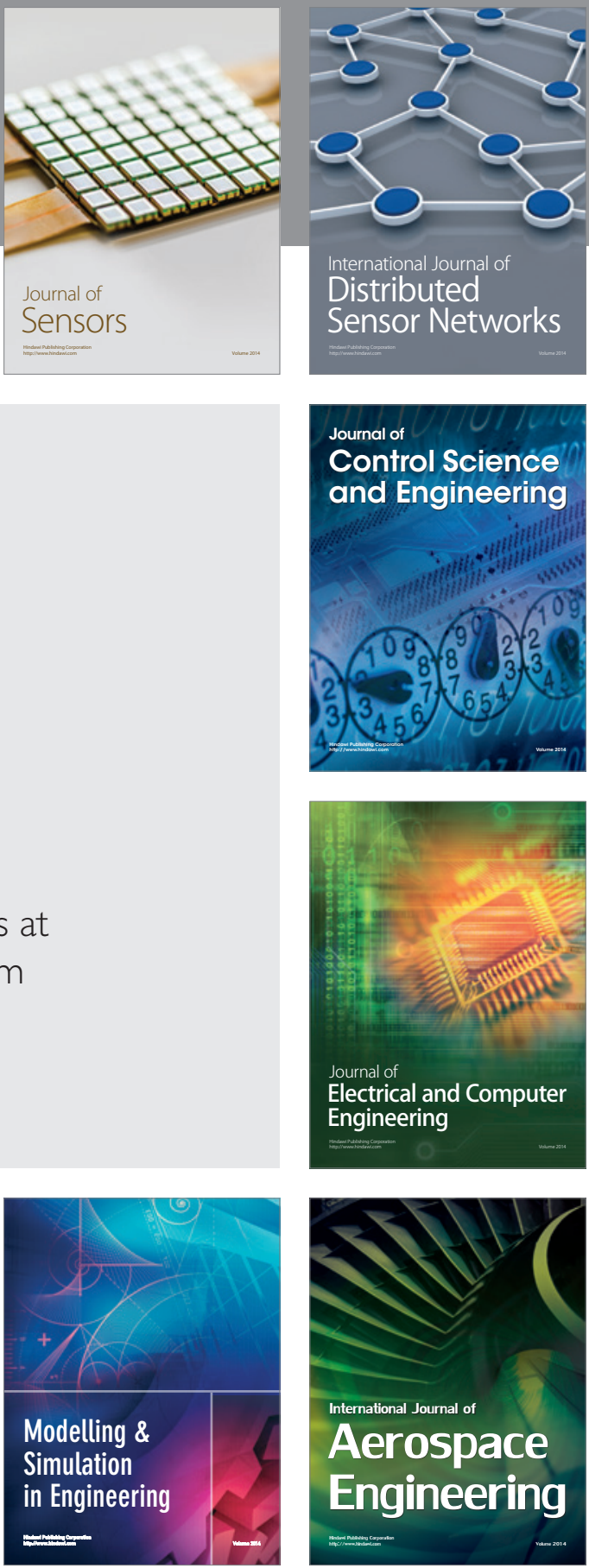

Journal of

Control Science

and Engineering
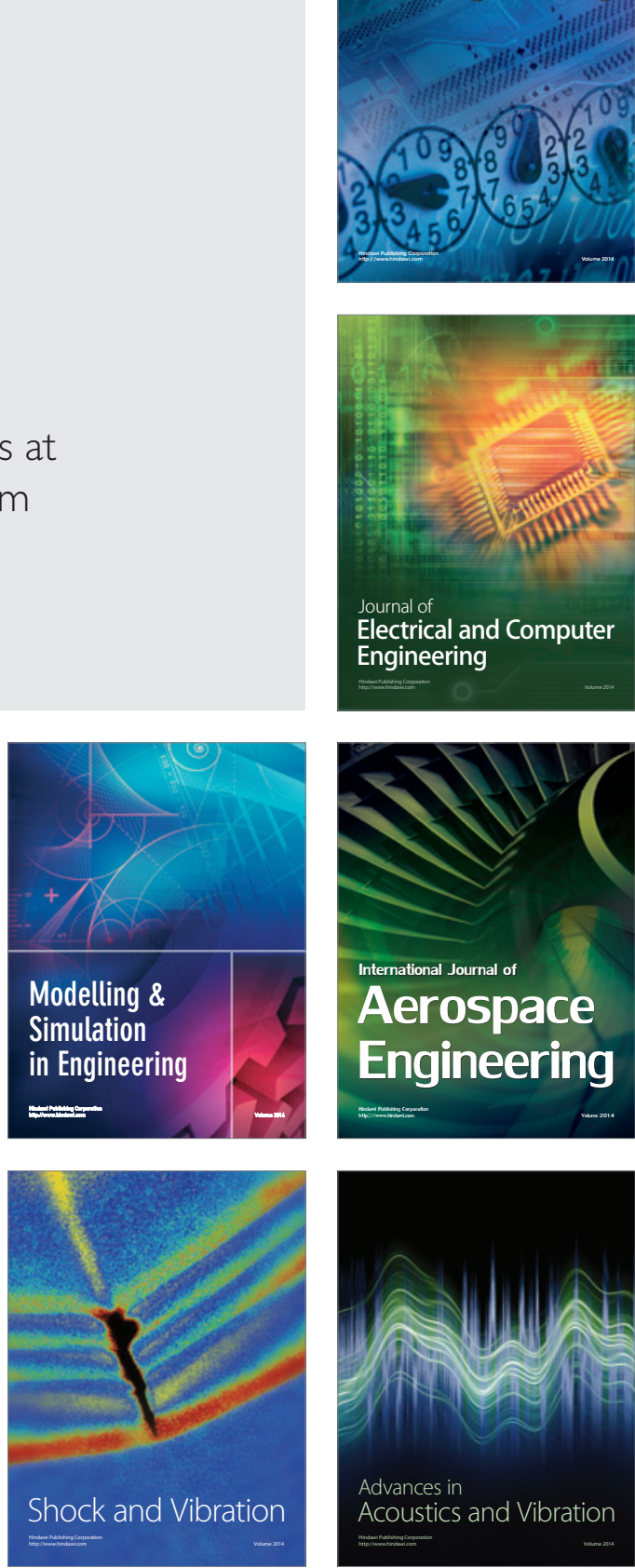